\title{
TURBULENT SHEAR FLOW DOWNSTREAM OF A SPHERE WITH AND WITHOUT AN O-RING LOCATED OVER A PLANE BOUNDARY
}

\author{
Muammer OZGOREN ${ }^{1}$, Abdulkerim OKBAZ ${ }^{2}$, Sercan DOGAN ${ }^{3}$, Besir SAHIN ${ }^{4}$, Huseyin AKILLI ${ }^{5}$
}

\begin{abstract}
Flow-structure interaction of separated shear flow from the sphere and a flat plate was investigated by using dye visualization and the particle image velocimetry technique. Later, a passive control method was applied with $2 \mathrm{~mm}$ oring located on the sphere surface at $55^{\circ}$ from front stagnation point. The experiments were carried out in open water channel for Reynolds number value of $R e=5000$. Flow characteristics have been examined in terms of the 2-D instantaneous and time-averaged velocity vectors, patterns of vorticity, streamlines, rms of velocity fluctuations and Reynolds stress variations and discussed from the point of flow physics, vortex formation, lengths of large-scale Karman Vortex Streets and Kelvin-Helmholtz vortices depending on the sphere locations over the flat plate. It is demonstrated that the gap flow occurring between the sphere bottom point and the flat plate surface has very high scouring effect until $h / d=0.25$ and then unsymmetrical flow structure of the wake region keeps up to $h / D=1.0$ for smooth sphere. For the sphere with o-ring, the wake flow structure becomes symmetrical at smaller gap ratios and reattachment point on the flat plate surface occurs earlier. Moreover, o-ring on the sphere diminishes peak magnitudes of the flow characteristics and thus it is expected that the flow-induced forces will be lessened both on the sphere and flat plate surface. Vortex formation lengths and maximum value occurring points become closer locations to the rear surface of the sphere with o-ring.
\end{abstract}

Keywords: boundary layer, o-ring, sphere, PIV, passive control, turbulence, vorticity, wake

\section{INTRODUCTION}

Flow around sphere has many engineering applications in single and two phase flows such as air pollution, nuclear and thermal power plants, towed sonars, swimming bodies in the water, pneumatic and hydraulic conveying, chemical and food processing, conveying of sediments in the river, rain drops, combustion systems and sport balls. The wake of a sphere in uniform unbounded flow condition has been studied extensively for several years because of its complex flow features and practical applications. In contrast, the turbulent shear flow downstream of a sphere in presence of a plane boundary has

\footnotetext{
${ }^{1}$ Muammer OZGOREN, Selcuk University, Mechanical Engineering Department, mozgoren@selcuk.edu.tr

${ }^{2}$ Abdulkerim OKBAZ, Selcuk University, Mechanical Engineering Department, abdulkerim_okbaz@hotmail.com

${ }^{3}$ Sercan DOGAN, Selcuk University, Mechanical Engineering Department, sercandogan@selcuk.edu.tr

${ }^{4}$ Besir SAHIN, Cukurova University, Mechanical Engineering Department, bsahin@cu.edu.tr

${ }^{5}$ Huseyin AKILLI, Cukurova University ,Mechanical Engineering Department, hakilli@cu.edu.tr
} 
received less attention and its passive control has not been studied in detail. In nature and engineering applications surfaces of most structures are exposed to non-uniform incoming boundary layer flow due to proximity of a wall. There are numerous practical cases in which spherical structures are immersed in a boundary layer flow. In the heat transfer enhancement and skin friction reduction applications spherical shaped bluff bodies can be used to manipulate boundary layer as surface modifications. When a sphere is immersed close to a wall, vortex shedding changes noticeably due to the influence of a non-uniform velocity profile which is formed as a result of boundary layer flow developed over the plane wall. This results in asymmetry in the strength of vortex shedding from upper and lower sides of the sphere and changes the direction of the mean force acting over the body away from the plane wall. Further, interactions of vortical structures shed from the sphere and the boundary layer flow result in complex flow structures depending on the gap between the sphere and plane wall. Thus, the turbulent wake of the sphere and plane boundary layer interaction is influenced by several factors, such as approaching boundary layer thickness, free-stream disturbances, Reynolds number and gap between the sphere and the plane wall and so that a wellunderstanding of this kind of flows have crucial importance to choose or develop suitable flow control methods.

Gad-el-hak (1996) pointed out that the ability to manipulate a flow field to effect a desired change is of immense practical importance. As a scientific discipline and as a technological curiosity, flow control is perhaps more hotly pursued by scientists and engineers than any other area in fluid mechanics. Flow control involves passive or active devices to effect a beneficial change in wall bounded or free-shear flows. Whether the task is to delay/advance transition, to suppress/enhance turbulence or to prevent/provoke separation, drag reduction, lift enhancement, mixing augmentation, heat transfer enhancement and flow-induced noise suppression. Passive control is applied by means of fixed or static devices such as roughness, splitter plate, trip wire, natural ventilation, streamlining the bluff body, helical strakes, perforated surface, ribbons, hemi spherical surface control bumps, spoiler adding and so on that modify the flow while active control is realized by dynamic actuators such as MEMS, small means of jets, acoustic transducers, suction etc. and requires some (ideally small) energy input [1].

A study related with interaction of boundary layer and sphere was performed by Tsutsiu (2008). He conducted experiments in wind tunnels to investigate flow around a sphere placed at various heights above a plane turbulent boundary layer at the Reynolds number $8.3 \times 10^{4}$ based on the sphere diameter. The flow was visualized by smoke-wire method and the surface oil-flow pattern method [2]. Jang and Lee (2008) reported the vortical flow structures of the sphere wake in the stream-wise plane at $\operatorname{Re}=11000$ in order to demonstrate flow structures and turbulence statistics. Their results could enhance understanding of the vortical flow structure and shear-layer instability inside the near-wake of a sphere [3].

It is well-known that in uncontrolled flows around a sphere, the drag coefficients rapidly decrease down to approximately 0.07 and this phenomenon is called the drag crisis occurred around $\operatorname{Re}>2 \times 10^{5}$ [4] (Achenbach, 1972). The cause of this rapid drag-coefficient reduction is the existence of small separation bubble(s) above the surface. Suryanarayana and Meier (1995) and Suryanarayana \& Prabhu (2000) investigated the ventilation effects on the flow characteristics in the wake of a sphere [5-6]. They recorded that the ventilation decreases the drag coefficient for Reynolds number higher than critical value while it is less effect on the flow structure at intermediate Reynolds numbers. At the critical Reynolds number, disturbances existing in the boundary layer 
rapidly grow along the separated shear layer, and high momentum fluids in the free stream are entrained toward the bluff-body surface. This causes the reattachment of the flow (thus forming a separation bubble above the surface) and generates strong nearwall momentum, resulting in the delay of main separation. The generation of strong near-wall momentum by early separation and reattachment is possible at the critical Reynolds number in uncontrolled flow [7] (Choi et al. 2008). Below the critical Reynolds numbers by using some surface disturbances such as o-ring it is possible to cause local flow separation and trigger the boundary layer flow from laminar to turbulent which retards the mean flow separation on the sphere. In order to decrease the drag coefficient of the sphere, some studies have been performed and practically applied. In the parametric study of Ozgoren et al (2011a) o-rings with $2 \mathrm{~mm}, 3 \mathrm{~mm}$ and $4 \mathrm{~mm}$ diameters are located at the front side of a sphere having $42.5 \mathrm{~mm}$ diameter at angles of $45^{\circ}, 50^{\circ}$, $55^{\circ}$ and $70^{\circ}$ at $\operatorname{Re}=5000$ to investigate the suppression effect of the o-rings on the flow structure. They found that the optimum angular position and diameter of the o-ring to passively control the flow structure is $55^{\circ}$ and $2 \mathrm{~mm}$ respectively [8]. An experimental investigation of flow structures downstream of a circular cylinder and sphere immersed in a free-stream flow was performed for $\mathrm{Re}=5000$ and 10000 using qualitative and quantitative flow visualization techniques by Ozgoren et al. (2011b). Flow data reveal that the size of wake flow region, the location of singular and double points, the peak values of turbulence quantities, such as Reynolds stress correlations, vorticity fluctuations and turbulent kinetic energy vary as a function of models' geometry and Reynolds Numbers. They stated that the concentration of small scale vortices is more dominant in the wake of the sphere than that of the cylinder [9]. Aoki et al (2003a and 2003b) studied on the flow structure around a sphere with dimples and flow pattern on surface structure of baseball. They stated that modified surface of sphere change flow patterns and aerodynamic characteristics of the wake [10-11].

In particular the sphere is regarded as a representative three-dimensional bluff body, and its wake structure is quite complex because of 3-D vortex shedding. Some of the other related studies on the flow control concerning with the present study under the uniform flow condition can be found in the literature [12-26] and cited therein.

In the present study, flow-structure interaction of separated shear flow from the sphere and a flat plate is investigated by using dye visualization and the particle image velocimetry (PIV) technique at $\mathrm{Re}=5000$. The sphere locations from the flat plate surface are varied in the range of $0 \leq G / D \leq 1.5$. Later, a passive control method is applied with 2 $\mathrm{mm}$ o-ring located on the front surface of the sphere at $55^{\circ}$ degree for the same positions.

\section{EXPERIMENTAL SETUP}

Experiments were performed in a large-scale open water channel with a test section length of $8000 \mathrm{~mm}$ and a width of $1000 \mathrm{~mm}$ at the Department of Mechanical Engineering at Cukurova University, Turkey. To perform the present experimental study, the test section made from $15 \mathrm{~mm}$ thick transparent Plexiglas sheet, which had a total height of $750 \mathrm{~mm}$, was filled with water to a level of only $450 \mathrm{~mm}$. Before reaching the test chamber, the water was pumped into a settling chamber and passed through a honeycomb section and a two-to-one channel contraction. An overview of experimental system of the sphere is shown in Figure 1a. Free stream turbulence intensity of the flow is less than $0.5 \%$ in the range of the present Reynolds numbers, $\operatorname{Re}=\left(U_{\infty} D\right) / v$, based on the sphere diameter (D). Here, $v$ and $D$ are kinematics viscosity and diameter of the sphere (D), respectively. $U_{\infty}$ is free stream velocity taken as $117.6 \mathrm{~mm} / \mathrm{s}$ for $R e=5000$. The sphere with a diameter of $42.5 \mathrm{~mm}$ was made of Plexiglas so that the laser light propagates easily from them. In addition, water cell segment of the sphere equator with a diameter of $38.5 \mathrm{~mm}$ and a wall thickness of $2.0 \mathrm{~mm}$ was created. It was filled with distilled water and had a total height of $8 \mathrm{~mm}$ in order to reduce largely the laser light 
deflection on the sphere. The sphere surface was highly polished to avoid effects of surface roughness. The laser sheet was located at $225 \mathrm{~mm}$ above the bottom surface of the channel while the water height $h_{w}$ was $450 \mathrm{~mm}$ in all cases. To support the sphere in the water channel, a circular bar with a $5 \mathrm{~mm}$ diameter was connected to the sphere from the top. Disturbance effect of the support bar on the laser sheet location of the measurement plane that was observed by dye injection was negligible in the consideration of support diameter with respect to the sphere diameter. The solid blockage ratio of the sphere including support was $1.3 \%$. Sphere models are presented in Figure $2 \mathrm{~b}$. The sphere with o-ring made of solid plexiglas and did not permit to pass the laser light. The o-rings with $2 \mathrm{~mm}$ diameter were made of rubber located at the front side of the sphere having $42.5 \mathrm{~mm}$ diameter at angle of $55^{\circ}$. A Flat plate with dimensions of $2000 \mathrm{~mm} \times 980 \mathrm{~mm} \times 10 \mathrm{~mm}$ having sharp leading edge is located on the bottom surface of water channel. The hydrodynamically developed boundary layer was obtained by a tripping wire of diameter $5 \mathrm{~mm}$ placed $80 \mathrm{~mm}$ downstream of the leading edge of the flat plate. The sphere model was located $1400 \mathrm{~mm}$ downstream of the leading edge of the flat plate from the centreline of the sphere. The gap between the lower point of the sphere and surface of the flat plate was changed from $0 \mathrm{~mm}$ to 63.75 $\mathrm{mm}$ and normalized with the sphere diameter designated as G/D. The obtained velocity profile with trip wire is shown in Figure 2. It is determined in absence of the sphere that the resulting hydrodynamically developed boundary layer thickness is $65 \mathrm{~mm}$ at the centre of the sphere location.

$\mathrm{Nd}$ :YAG laser was used to generate a laser sheet that was perpendicular to the axis for the sphere and the symmetry axis (i.e. equator of the sphere) was passed through them. A CCD camera having a resolution of $1,600 \times 1,186$ pixels was used to record the images. The seeding particles with a diameter of $10 \mu \mathrm{m}$ in the flow were silver metallic coated hollow plastic spheres. The densities of the particles and water are close enough so that the distribution of particles in suspension remains uniform for several hours. The high-image-density criterion was satisfied by ensuring that a minimum of approximately 20-30 particles was contained within the interrogation area. The illuminating laser sheet thickness in the flow field was approximately $1.5 \mathrm{~mm}$. As shown in Figure 1, the camera was mounted in a fixed position beneath the water tank. Dantec Flow Grabber digital PIV software employing the cross-correlation algorithm was used to compute the raw displacement vector field from the particle image data.

An interrogation window of $32 \times 32$ pixels in the image was selected and converted to grid size approximately $1.44 \times 1.44 \mathrm{~mm}^{2}$ for the single sphere $(0.034 \mathrm{D} \times 0.034 \mathrm{D})$. The overall fields of physical view were for both spheres, yielding to 7,227 (99x73) velocity vectors for whole taken images. During the interrogation process, an overlap of $50 \%$ was employed in order to satisfy the Nyquist criterion. Patterns of instantaneous particle images with a total of 350 images for a continuous series were taken at the rate of 15 $\mathrm{Hz}$, thereby spanning $23.27 \mathrm{sec}$. Averaged patterns of the flow structure were calculated from all of the instantaneous images. The laser sheet was generated from a dual pulsed $\mathrm{Nd}$ :YAG system, having the maximum output of $120 \mathrm{~mJ}$ per pulse, which had time delays $\Delta t=1.0-1.7 \mathrm{~ms}$ for the present experiments. Inappropriate displacement vectors caused by shadows, reflections, or laser sheet distortions in the flow field replaced by using bilinear interpolation between surrounding vectors in the post-processing step. This algorithm included magnification factor and image captured rate to calculate velocities from the valid vectors. The field was then smoothed by a Gaussian weighted averaging technique. To minimize distortion of the velocity field, a smoothing parameter of 1.3 was chosen. After having been vector field, the vorticity patterns of the wake flow were determined from the velocity field using an in-house software. 
SIDE VIEW

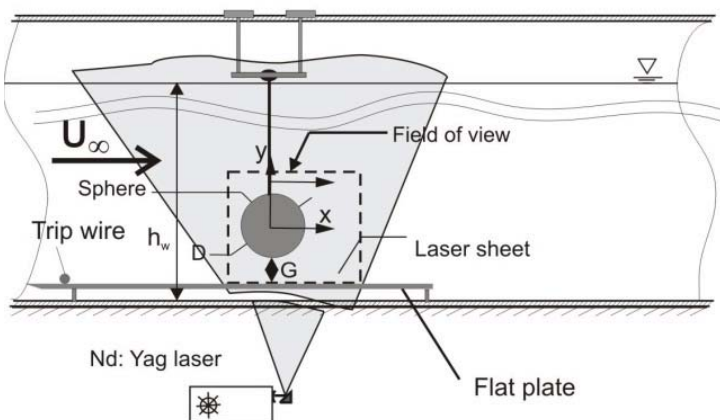

BACK VIEW

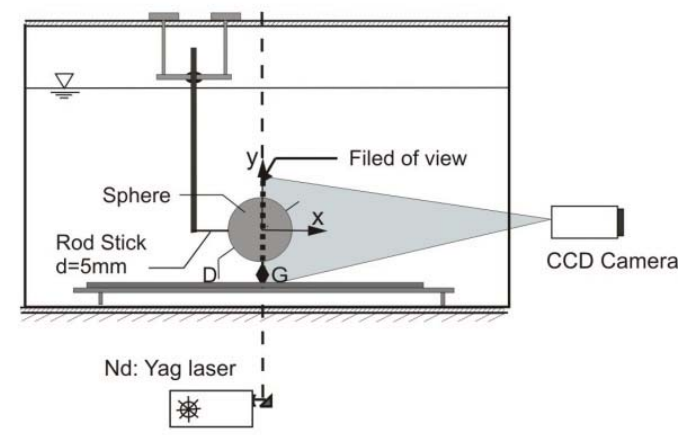

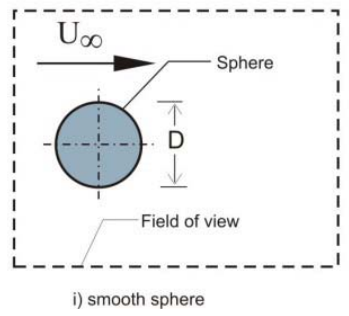

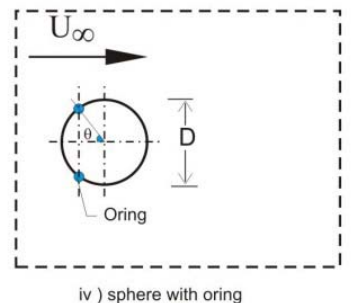

Figure 1: Schematic view of the experimental setup of PIV system and sphere position for a smooth sphere and sphere with o-ring case

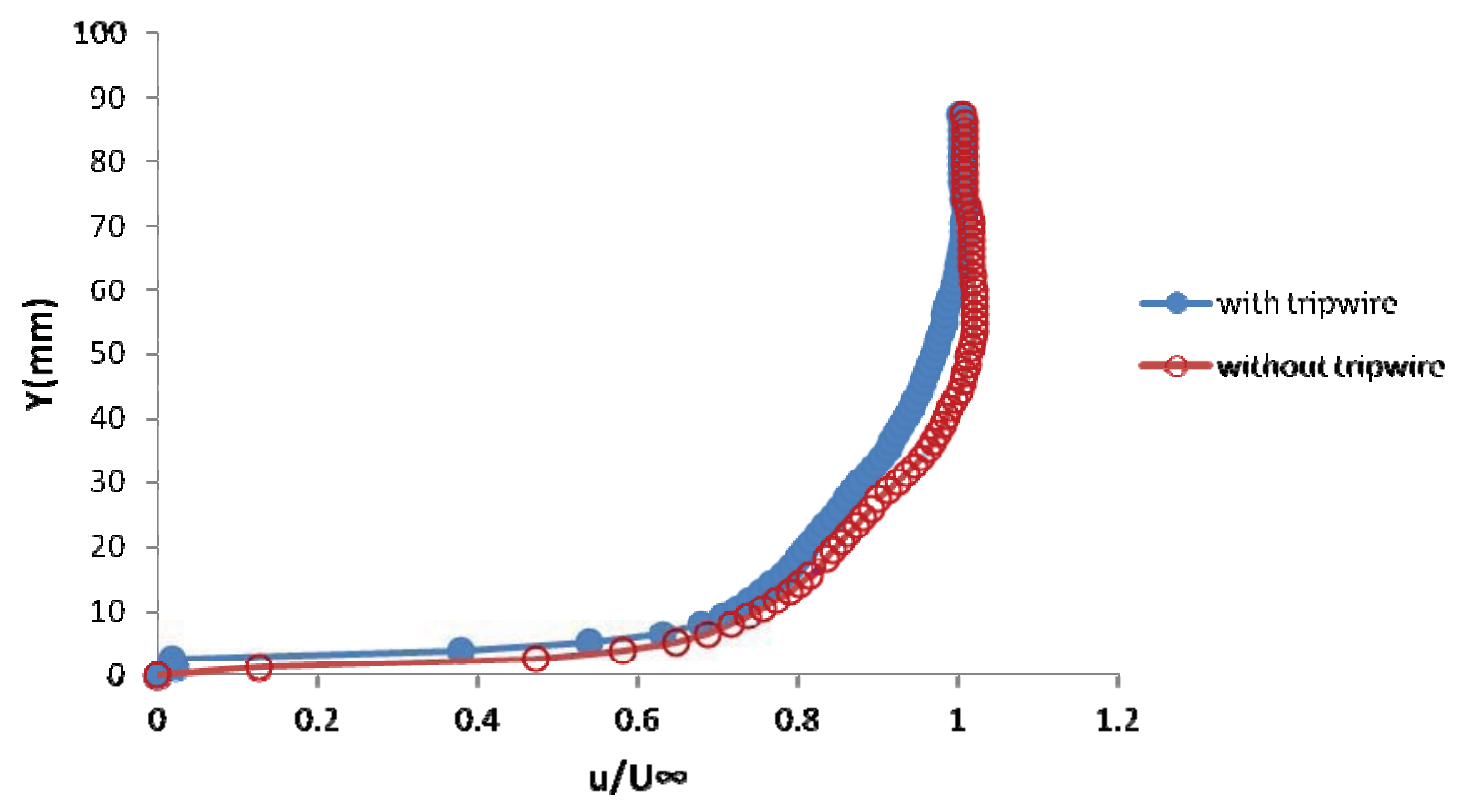

Figure 2: Velocity profiles at the location of flow field over the flat plate with trip wire

\section{RESULTS AND DISCUSSION}

Figure 3 shows comparison of flow visualization images of instantaneous flow fields with laser illumination of Rhodamine dye injection technique around the smooth sphere (left column) and sphere with $2 \mathrm{~mm}$ o-ring (right column) located over a flat plate for 
different gap ratios at $\mathrm{Re}=5000$. In the dye visualization representative images the small scale vortices are designated by $A$ to $F$ to show evolution and progress of them. The separated and recirculating flow in the near-wake region with the help of visualizing are clearly seen for the sphere with laser illumination using the Rhodamine dye injection technique in the near-wake region.

For $G / D=0$, the flow separates from only upper surface of the sphere in the visualization plane and small scale vortices in the wake region are formed around larger vortices with a wavy appearance due to Kelvin Helmholtz instability in the boundary layer. As the flow travels in the downstream direction, the dimensions of the vortices increase then these vortices are shed from the upper shoulder of the sphere directly to the inward wake region and the separated flow reattaches on the flat plate. In the case of $G / D=0.1$, the gap flow between the bottom of the sphere and the flat plate highly affects the wakes of both smooth and passive controlled spheres. The wake regions of both smooth and controlled sphere by $2 \mathrm{~mm}$ o-ring move upward direction from the plane wall. For an increased gap ratio $(G / D=0.25)$, as clearly seen from the Figure 3 , there is still a tendency to move upward of smooth sphere wake whereas the controlled sphere wake moves downward due to the delayed flow separation from upper side of the sphere.

As the gap ratio increases $(G / D \geq 0.50)$ the effect of gap flow between lower point of the sphere and the flat plate surface loses in both smooth and controlled sphere cases. Moreover, Karman vortex streets begin to form and appear symmetrical in the downstream of the smooth sphere. On the other hand, these structures appear highly suppressed by the effect of o-ring for controlled sphere.

To visualize and compare the vortex dynamics in the effect of proximity of the wall and non-uniform velocity profile of boundary layer, comparison of instantaneous velocity fields $\mathrm{V}$ and vorticity contours $\omega^{*}$ around the smooth sphere and the sphere with $2 \mathrm{~mm}$ o-ring located over a flat plate for different gap ratios at $\mathrm{Re}=5000$ are presented in Figure 4 and and Figure 5, respectively. Instantaneous vorticity contours of the wake structure is normalized as $\omega^{*}$ (i.e., $\omega^{*}=\omega \mathrm{D} / \mathrm{U}_{\infty}$ ). All figure dimensions are normalized with the sphere diameter designated as $x / D, y / D$ and $G / D$.

When the sphere is located on the flat plate $(G / D=0)$, for both smooth and controlled sphere cases, the contact point of the sphere and the flat plate blocks the fluid flow resulting in preventing the shedding of vortices from the lower side of the sphere. Thus, only negative vortices shed from upper side of the sphere in the visualization plane. For $G / D=0.1$ both shear layers develop as elongated chains of vortices. The vortices produced from the flow separation around the periphery of the sphere have a tendency to move inwards because of the lower pressures prevailing within the wake. In the case of smooth sphere the gap flow is more effective than that of the controlled sphere and shedding vortices are conveyed through the gap flow along the base of the smooth sphere. On the other hand, as seen from instantaneous velocity fields $V$ (Figure 4) and vorticity contours $\omega^{*}$ (Figure 5 ) the $2 \mathrm{~mm}$ o-ring located on the front side of the sphere at $55^{\circ}$ angle modifies the gap flow and direction of the gap flow becomes parallel to the flat plate. For the low gap ratios $(G / D=0.1$ and 0.25$)$, owing to the velocity profile of the bounded gap flow between the sphere and the flat plate negative vortices also form. 


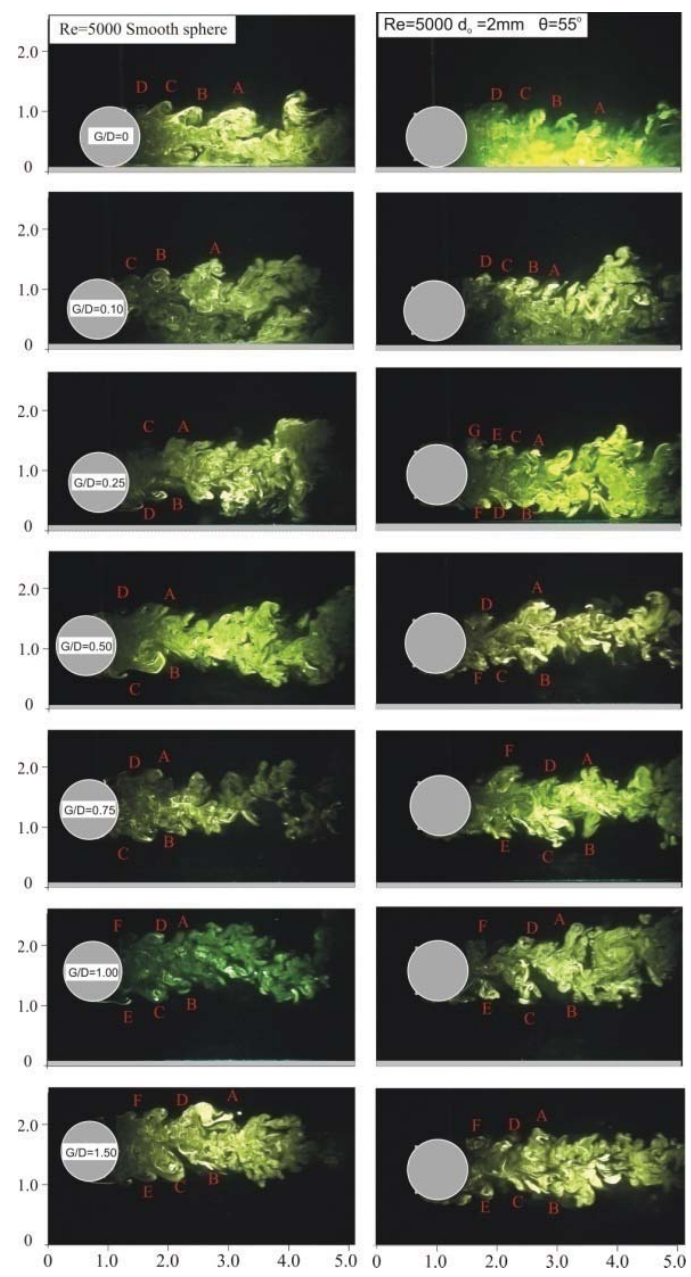

Figure 3: Comparison of flow visualization of flow structure with laser illumination of Rhodamine dye injection technique around the smooth sphere (left column) and sphere with $2 \mathrm{~mm}$ o-ring (right column) for $\mathrm{Re}=5000$.

Because of the difference in celerity of the positive vortex shed from the lower side of the sphere than that of the negative vortex shed from the upper side, vorticity peaks of the lower side is smaller than that for the upper side. The stream-wise separation of successive vorticity peaks in the near wake region for the sphere with o-ring are smaller than those of smooth sphere case for all gap ratios.

Figures 6 and 7 present the time-averaged velocity vectors $\langle\mathrm{V}\rangle$ and corresponding patterns of streamline $\langle\psi\rangle$, respectively. These figures reveal information about size of the wake regions of the smooth and passively controlled spheres located over the flat plate for a well comparison. It is clearly seen that the presence of the flat late significantly affects the velocity field for small values of gap ratio. For the case of the sphere touching the flat plate, a large circulation region which is entrapped by shear layer separated from upper side of the sphere forms. For $G / D=0$, the separated flow from the upper side of the smooth sphere reattaches on the flat plate after approximately $1.42 \mathrm{D}$ away from the base of the sphere in the downstream direction while it reattaches on the flat plate after approximately $0.92 \mathrm{D}$ for the sphere controlled by $2 \mathrm{~mm}$ o-ring. 
EPJ Web of Conferences
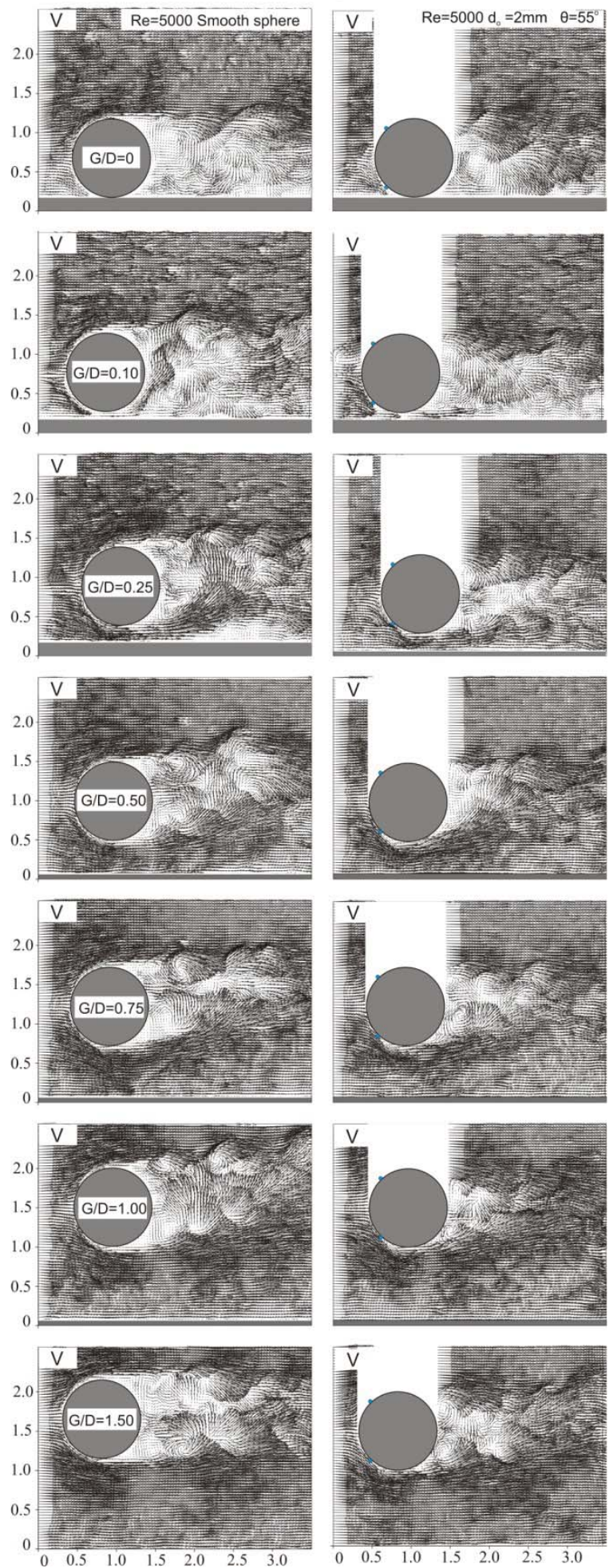

Figure 4: Variation of the instantaneous velocity fields $V$ depending on the sphere locations over the flat plate and effect of $2 \mathrm{~mm}$ o-ring for $\mathrm{Re}=5000$.
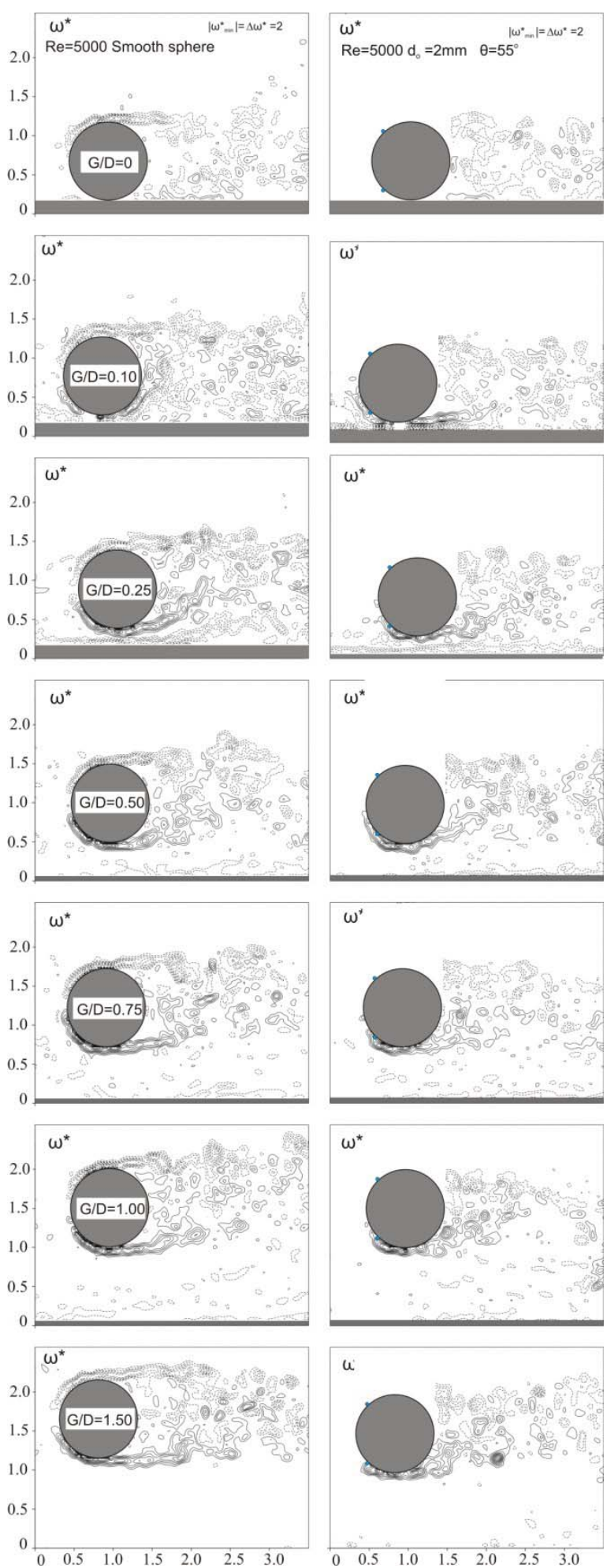

Figure 5: Variation of the instantaneous vorticity contours $\omega^{*}$ depending on the sphere locations over the flat plate and effect of $2 \mathrm{~mm}$ 

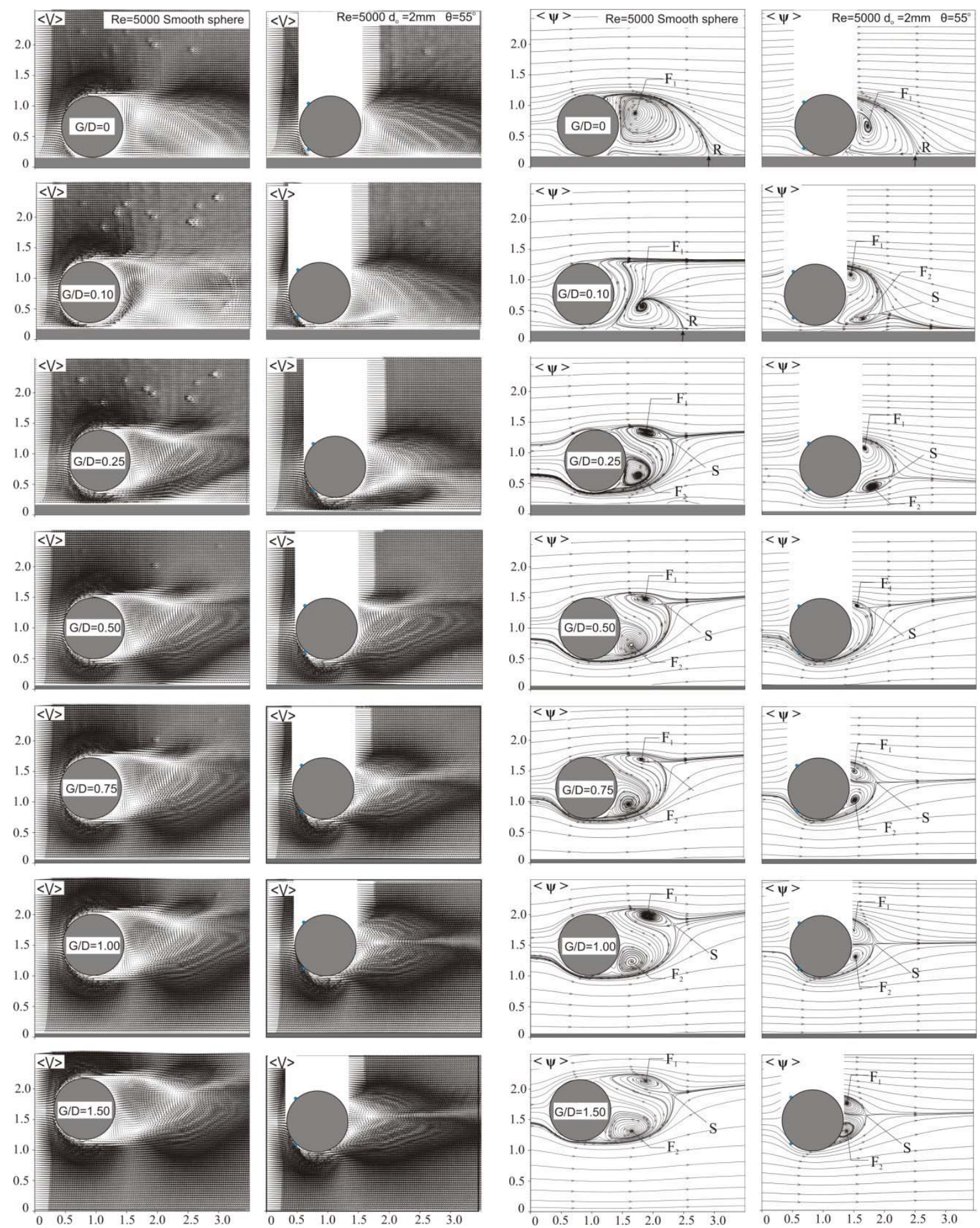

Figure 6: Variation of the time-averaged velocity field $\langle\mathrm{V}\rangle$ depending on the sphere locations over the flat plate and effect of $2 \mathrm{~mm}$ o-ring for $\mathrm{Re}=5000$.
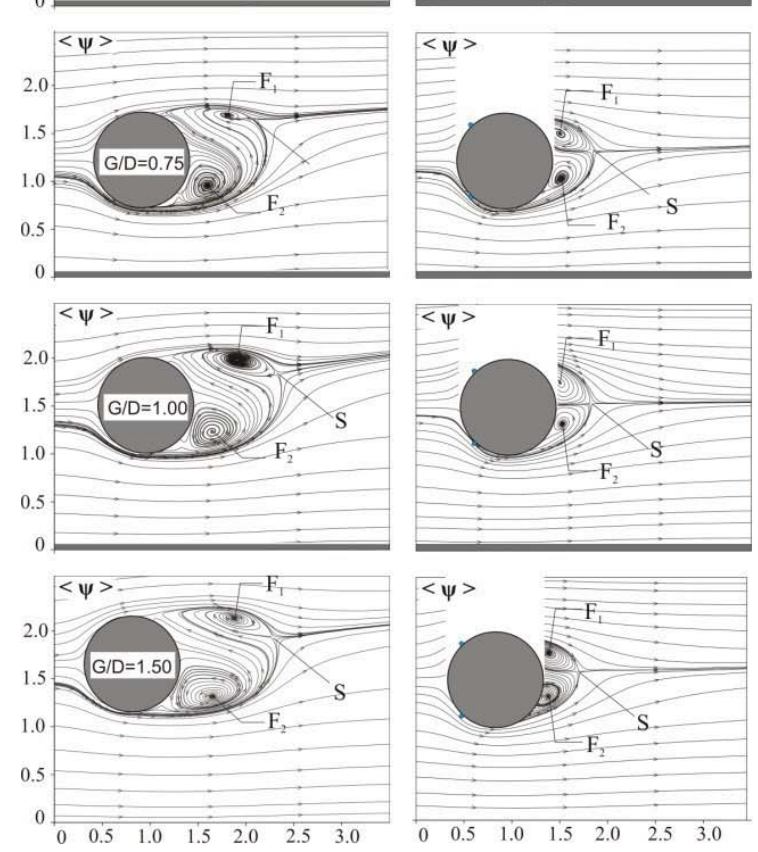

Figure 7: Variation of the streamline patterns $\langle\psi\rangle$ depending on the sphere locations over the flat plate and effect of $2 \mathrm{~mm}$ o-ring for $\mathrm{Re}=5000$. 
As the gap ratio increases to $G / D=0.1$, flow patterns for both sphere cases significantly change in a different way. For the smooth sphere case, as seen from the streamline patterns (Figure 7), a large separation bubble is formed and as seen from the timeaveraged velocity vectors (Figure 6) the flow in this region is relatively slow. Moreover, the fluid flow through the small gap turns sharply through the intermediate region between the base of the sphere and the large separation bubble, subsequently interacting with the fluid separated from the upper side of sphere. This interaction affects the vortex shedding characteristics and creates a good mixing of fluid layers causing enhancement in the wake, which is helpful for convective phenomena. In addition, no combined recirculation region in the wake occurs owing to Coanda effect. That is, the flow pass the sphere does not shed from the periphery of the sphere due to powerful jetlike flow between the sphere and flat sphere. For the higher gap ratios ( $G / D \geq 0.25)$ the two focuses $F_{1}$ and $F_{2}$ in the recirculation wake region happen and they are asymmetric about the centreline of the smooth sphere. The lower recirculation region is larger than the upper one due to the gap flow. Also, this is the result of the non-uniform velocity profile in the boundary layer.

In contrast to the smooth sphere case, there is a deflection in the wake region of the controlled sphere with an o-ring through to downward direction for $G / D=0.1$ and 0.25 (Figure 7). This situation changes the direction of the mean force acting on the spherical body downward to the flat plate. Additionally, two recirculation regions are formed on the both upper and lower sides of the controlled sphere with different lengths in the stream wise direction. For $G / D=0.5$ the direction of the controlled sphere wake region moves upwards away from the wall and only one recirculation region is formed. For larger gap ratios $(G / D \geq 0.5)$ the streamline patterns form two recirculation regions which are reasonably symmetric about the centreline of the controlled sphere similar to the case of uniform approaching flow conditions. Detailed information of saddle point locations for smooth and sphere with o-ring with $2 \mathrm{~mm}$ are compared in Table 1.

Table 1: comparison of the saddle point location for the two sphere models.

\begin{tabular}{|l|c|c|}
\hline $\begin{array}{l}\text { Gap } \\
\text { ratios }\end{array}$ & $\begin{array}{c}\text { Saddle points of the Smooth } \\
\text { sphere }\end{array}$ & $\begin{array}{c}\text { saddle points of the sphere with } \\
2 \mathrm{~mm} \text { o-ring at } 55^{\circ}\end{array}$ \\
\hline $\mathrm{G} / \mathrm{D}=0$ & $\mathrm{~L} / \mathrm{D}=1.419$ & $\mathrm{~L} / \mathrm{D}=0.925$ \\
\hline $\mathrm{G} / \mathrm{D}=0.10$ & $\mathrm{~L} / \mathrm{D}=1.075$ & $\mathrm{~L} / \mathrm{D}=0.518$ \\
\hline $\mathrm{G} / \mathrm{D}=0.25$ & $\mathrm{~L} / \mathrm{D}=0.659$ & $\mathrm{~L} / \mathrm{D}=0.471$ \\
\hline $\mathrm{G} / \mathrm{D}=0.50$ & $\mathrm{~L} / \mathrm{D}=0.674$ & $\mathrm{~L} / \mathrm{D}=0.235$ \\
\hline $\mathrm{G} / \mathrm{D}=0.75$ & $\mathrm{~L} / \mathrm{D}=0.613$ & $\mathrm{~L} / \mathrm{D}=0.400$ \\
\hline $\mathrm{G} / \mathrm{D}=1.00$ & $\mathrm{~L} / \mathrm{D}=0.845$ & $\mathrm{~L} / \mathrm{D}=0.344$ \\
\hline $\mathrm{G} / \mathrm{D}=1.50$ & $\mathrm{~L} / \mathrm{D}=1.001$ & $\mathrm{~L} / \mathrm{D}=0.353$ \\
\hline
\end{tabular}

For the sphere with o-ring, the wake flow structure becomes symmetrical at smaller gap ratios and reattachment point on the flat plate surface occurs earlier. It is demonstrated from the obtained results that the flow structure in wake region of the sphere with $2 \mathrm{~mm}$ o-ring at $55^{\circ}$ is significantly modified by passive control application and hence the distance between the sphere base and saddle points decreases as seen from timeaveraged flow patterns in Figures 6 and 7 as well as in Table 1 . Therefore, it can be stated that the sphere with $2 \mathrm{~mm}$ o-ring at $55^{\circ}$ triggers the flow from laminar to turbulence at low Reynolds number such as $\mathrm{Re}=5000$ which retards the flow separation on the sphere. 
Figure 8 shows variation of the time-averaged stream-wise velocity fluctuations $\left.<\mathrm{u}_{\mathrm{rms}} / \mathrm{U}_{\infty}\right\rangle$ depending on the sphere locations over the flat plate and effect of $2 \mathrm{~mm}$ o-ring at $R e=5000$. A global view on the asymmetry in the intensity of the time-averaged stream-wise velocity fluctuations show a thinning of the lower shear layer and a thickening of the upper shear layer due to the influence of boundary layer developed over the flat plate. However, for the case $G / D=0.25$ the stream-wise velocity fluctuations of the lower shear layer of the controlled sphere is larger than those of the upper one because of the gap flow. It is obtained from the comparison of the smooth sphere and sphere with o-ring that the maximum point locations of the rms velocities in streamwise direction occur closer to the sphere base for the sphere with o-ring case while their magnitudes decrease. For $G / D=0$ only stream-wise velocity fluctuations of the upper shear layer are formed for both smooth and controlled sphere cases. Because of the wake flow structure of the controlled sphere becomes symmetrical at smaller gap ratios, the peak value of the stream-wise velocity fluctuation of the lower shear layer of the controlled sphere is larger than that of the smooth sphere for $G / D=0.50$. For higher gap ratios flow structure seems to be symmetrical for the case of sphere with o-ring.

To reveal the relative effects of G/D ratio on the wake of the smooth sphere and sphere with $2 \mathrm{~mm}$ o-ring and boundary layer interactions, Reynolds stress correlations $\left\langle\mathrm{u}^{\prime} \mathrm{v}^{\prime} / \mathrm{U}_{\infty}^{2}\right\rangle$ behind the smooth sphere (left column) and sphere with $2 \mathrm{~mm}$ o-ring (right column) are presented in Figure 9. In uniform approaching flow condition, Reynolds stress correlations of flow past a sphere are formed symmetric about the centreline of the sphere wake with negative and positive peak magnitudes. For $G / D=0$, only negative Reynolds stress occur on the upper shear layer for both spheres. Reynolds stress patterns produce the maximum Reynolds stress region closer to the base of the sphere with $2 \mathrm{~mm}$ o-ring at $55^{\circ}$ than that of the smooth sphere, although their magnitudes do not differ considerably. For the case $G / D=0.1$ besides the large-scale cluster of $\left\langle u^{\prime} v^{\prime} / U_{\infty}^{2}\right\rangle$ with negative value, there consist two additional small-scale clusters with positive value located downstream of the smooth sphere base, whereas there consists only one additional cluster of $\left\langle\mathrm{u}^{\prime} \mathrm{v}^{\prime} / \mathrm{U}_{\infty}^{2}\right\rangle$ with positive value located downstream of the sphere with $2 \mathrm{~mm}$ o-ring at $55^{\circ}$. This situation occurs as a result of the gap flow which is more dominant for the smooth sphere than that of the controlled sphere. As the gap ratio increases above the $G / D=0.10$ the distributions of $\left\langle\mathrm{u}^{\prime} \mathrm{v}^{\prime} / \mathrm{U}_{\infty}^{2}\right\rangle$ become asymmetric about the smooth sphere centreline with negative Reynolds stress in the upper shear layer and low levels of positive Reynolds stress in the lower shear layer.

For $G / D=0.1$ the mean flow patterns does not become symmetrical (Figure 7), however the peak magnitudes of the Reynolds stresses of the upper and the lower shear layers of the controlled sphere are close to each other. For $G / D=0.25$ the upper and lower shear layers of the controlled sphere become stronger comparing with the case $G / D=0.1$ and the distributions of $\left\langle\mathrm{u}^{\prime} \mathrm{v}^{\prime} / \mathrm{U}_{\infty}^{2}\right\rangle$ become more symmetric by getting equal approximately at the peak values of \pm 0.03 . When the gap ratio increases to $G / D=0.50$ the direction of the controlled sphere wake moves upward and the upper shear layer of the controlled sphere becomes stronger resulting an increase of the $\left\langle\mathrm{u}^{\prime} \mathrm{v}^{\prime} / \mathrm{U}_{\infty}^{2}\right\rangle$ magnitude to -0.042 while lower one's decreases to 0.022 . For $G / D=0.75,1.00$ and 1.50 the magnitudes of $\left\langle\mathrm{u}^{\prime} \mathrm{v}^{\prime} / \mathrm{U}_{\infty}^{2}\right\rangle$ peak values of the upper and lower shear layer of the controlled sphere become nearly equal as displayed in Figure 9. 


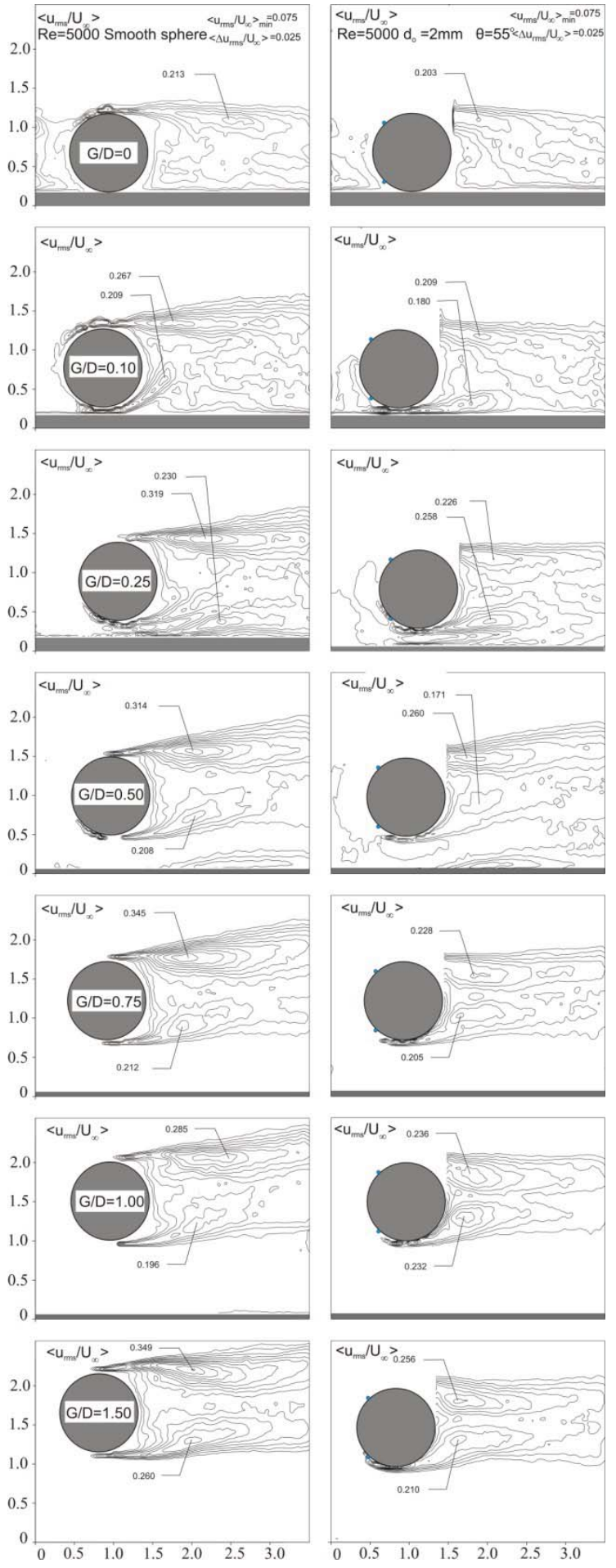

Figure 8: Variation of the time-averaged streamwise velocity fluctuations $<$ urms/Uळ> depending on the sphere locations over the flat plate and effect of $2 \mathrm{~mm}$ o-ring for $\mathrm{Re}=5000$.
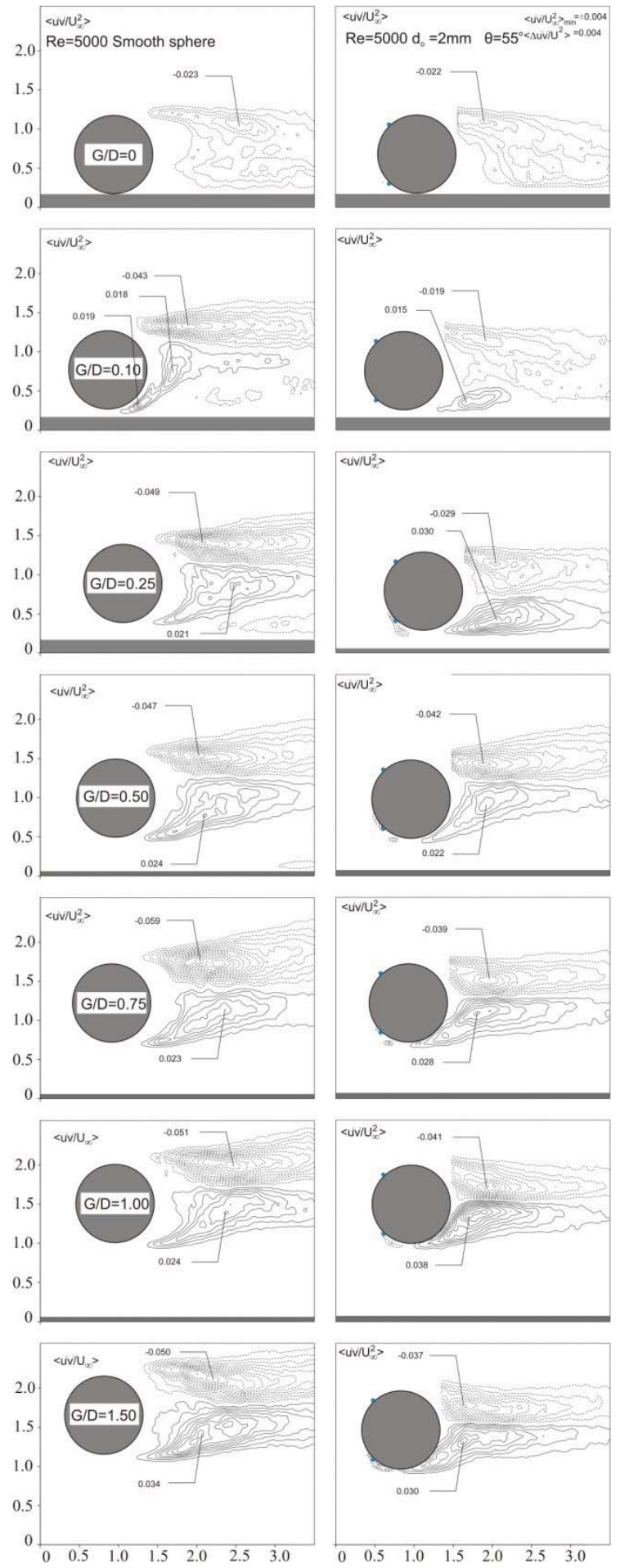

Figure 9: Variation of the Reynolds stress correlations $\left\langle\mathrm{u}^{\prime} \mathrm{v}^{\prime} / \mathrm{U}_{\infty}^{2}\right\rangle$ depending on the sphere locations over the flat plate and effect of $2 \mathrm{~mm}$ o-ring for $\mathrm{Re}=5000$. 


\section{CONCLUSIONS}

Experiments were conducted to investigate interaction of the wake region of a smooth sphere and sphere with $2 \mathrm{~mm}$ o-ring located at the front surface at angle of $55^{\circ}$ and hydrodynamically developed boundary layer over a flat plate in a large-scale open water channel via PIV and Rhodamine 6G visualization methods. The obtained main results of this study are summarized as follows.

1) $G / D$ ratio has a strong influence on the flow structure of the wake-boundary layer interaction for the smooth sphere and the sphere with $2 \mathrm{~mm}$ o-ring.

2) The non-uniform velocity profile of the boundary layer flow causes a difference in the strength of the separated shear layers from the periphery of the sphere.

3) For $G / D=0$ the shear layer separates from only upper sides of the both spheres in the visualization plane. Reattachment points for the smooth and sphere with o-ring $2 \mathrm{~mm}$ are determined as $1.42 \mathrm{D}$ and $0.92 \mathrm{D}$, respectively.

4) For $G / D=0.1$, the jet-like flow through the small gap turns sharply into the intermediate region between the rear of the smooth sphere and the large separation bubble and then it interacts with the fluid separated from the upper side of the smooth sphere. This interaction affects the vortex shedding characteristics and creates a good flow mixing resulting in an heat transfer enhancement.

5) For $G / D \geq 0.1$ the direction of the wake region of the smooth sphere is deflected to upward from the flat plate whereas there is a deflection in the wake region of the controlled sphere with $2 \mathrm{~mm}$ o-ring through to downward direction for $\mathrm{G} / \mathrm{D}=0.1$ and 0.25 . For $G / D=0.5$ the wake region of the controlled sphere with a $2 \mathrm{~mm}$ o-ring moves upward direction and for larger values of gap ratio they become symmetric about the sphere centreline.

6) It can be commented that saddle point locations for the sphere with $2 \mathrm{~mm}$ o-ring become closer to the rear surface of the sphere which is clear evidence of passive control of the flow structure, positively.

Finally, the obtained results for specially smooth sphere case might be used for validation of numerical studies.

\section{ACKNOWLEDGMENT}

The authors would like to acknowledge the funding of the Scientific and Technological Research Council of Turkey (TÜBİTAK) under contract no:109R028, Coordinatorship of Selcuk University's Scientific Research and Scientific Research Projects Office of Cukurova University under contract No: AAP20025. This study is prepared from Master of Science Thesis of Abdulkerim OKBAZ.

\section{RefEREnCES}

[1] Gad-el-Hak M.: Modern Developments in Flow Control, Applied Mechanics Review, vol, V. 49, 1996, pp.365-379.

[2] Tsutsui T.: Flow around a sphere in a plane turbulent boundary layer, Journal of Wind Engineering and Industrial Aerodynamics, 2008, 96, 779-792.

[3] Jang Y. II, J., Lee S.J.: PIV analysis of near-wake behind a sphere at a subcritical Reynolds number, Exp. Fluids. 44, 2008, Issue 6, 905-914.

[4] Achenbach E.: Experiments on the flow past spheres at very high Reynolds numbers, J. Fluid Mech., 1972, 54, 565-75. 
[5] Suryanarayana G. K., Meier GEA: Effect of ventilation on the flow field around a sphere, Exp Fluids 19, 1995, pp.78-88.

[6] Suryanarayana G. K., Prabhu A.: Effect of natural ventilation on the boundary layer separation and near-wake vortex shedding characteristics of a sphere, Exp. Fluids 29, Issue.7, 2000, pp.582-591.

[7] Choi H., Jeon W.P., Kim J.: Control of Flow Over a Bluff Body, Annu. Rev. Fluid Mech., 2008, 40, 113-139.

[8] Ozgoren M., Okbaz A., Dogan S., Kahraman A., Hassanzadeh R., Sahin B., Akilli H.: Passive Control of Vortical Flow Structure around a Sphere by an O-ring, Elazıg, $6^{\text {th }}$ International Advanced Technologies Symposium, 2011a, ETE-53.

[9] Ozgoren M., Pinar E., Sahin B., Akilli H.: Comparison of flow structures in the downstream region of a cylinder and sphere. Int. J. Heat Fluid Flow, 2011b, doi:10.1016/j.ijheatfluidflow.2011.08.003.

[10] Aoki K., Ohike A., Yamaguchi K., Nakayama Y.: Flying Characteristics and Flow Pattern of A Sphere With Dimples, Journal of Visualization, 2003a, pp.67-76.

[11] Aoki K., Kinoshita, Y., Nagase J., Nakayama Y.: Dependence of Aerodynamic Characteristics and Flow Pattern on Surface Structure of a Baseball, Journal of Visualization, 2003b, vol.6, no.2, pp.185-193.

[12] Rashidi M., Hetsroni G., Banarjee S.: Particle-turbulance interaction in a boundary layer, Int. J. Multiphase Flow, vol.16, no.6, pp.935-949, 1990.

[13] Bearman P., Brankovi'c M.: Experimental studies of passive control of vortexinduced vibration, European Journal of Mechanics B/Fluids 23, 2004, 9-15.

[14] Bearman P.W.: The effect of base bleed on the flow behind a two-dimensional model with a blunt trailing edge, The Aero. Ouarterly XVIII, 1967, 207-223.

[15] Jeon J., Choi J., Woo-Pyung J., Haecheon C., Jınıl P.: Active control of flow over a sphere for Drag Reduction at a Subcritical Reynolds number, J. Fluid Mech. vol.517, 2004, pp.113-129.

[16] Jeon S., Choi H.: Suboptimal Feedback Control for Drag Reduction in Flow over a Sphere, Bull. Am. Pbys. Soc., 2005, 50:56.

[17] Dandy D. S., Dwyer H. A.: A sphere in shear flow at finite Reynolds number: effect of shear on particle lift, drag, and heat transfer, J. Fluid Mech 216, 1990, pp.381-410.

[18] Owen J. C., Bearman P. W.: Passive control of VIV with drag reduction, Journal of Fluids and Structures, 15, 2001, pp.597-605.

[19] Haecheon C., Woo-Pyung J., Jinsung K.: Control of Flow Over a Bluff Body, Annu. Rev. Fluid Mech., 40, 2008, pp.113-39.

[20] Hadzic I., Bakic V., Peric M., Sajn V.: Kosel F.: Experimental and numerical studies of flow around sphere at sub-critical Reynolds number, Eng .Turbul. Model Exp. 5, 2002, pp.667-676,.

[21] Kim D. Yung, Choi H.: Vortical structures behind a sphere at subcritical Reynolds numbers, Phys Fluids 18(1):015102, 2006, doi:10.1063/1.2166454,.

[22] Leder A., Geropp D.: The unsteady flow structure in the wake of the sphere, SPIE 2052, 1993, pp.119-126.

[23] Lima H.-C., Leeb S.-J.: Flow control of a circular cylinder with O-rings, Fluid Dynamics Research 35, 2004, pp.107-122.

[24] Mehta D.: Aerodaynamic of Sport Balls, Ann. Rev. Fluid Mechs, 1985, Vol. , 17:151-89.

[25] Ozgoren M., Okbaz A., Kahraman A., Hassanzadeh R., Sahin B., Akilli H., Dogan S.: Experimental Investigation of the Flow Structure around a Sphere and Its Control with Jet Flow via PIV, Elazıg, 6th International Advanced Technologies Symposium, 2011c, ETE-50. 